\title{
A Significant Discrepancy Between Endocrinological and Clinical Phenotype and Immunological Phenotype in Autoimmune Polyglandular Syndrome Type 3
}

\author{
Hidetaka Hamasaki ${ }^{\mathrm{a}}$, Hidekatsu Yanaia, b, c, Chika Hiraishi ${ }^{\mathrm{a}}$, Sumie Moriyama ${ }^{\mathrm{a}}$
}

\begin{abstract}
Autoimmune polyglandular syndrome (APS) is made up of a group of autoimmune disorders of the endocrine glands. In APS type 3 , autoimmune thyroiditis occurs with another organ-specific autoimmune disease. APS type 3 is also associated with systemic autoimmune diseases such as sarcoidosis, Sjögren's syndrome and rheumatoid arthritis. We describe a 53-year-old woman with APS type 3 who showed a significant discrepancy between endocrinological and clinical phenotype and immunological phenotype.
\end{abstract}

Keywords: Autoimmune thyroiditis; Autoimmune polyglandular syndrome; Rheumatoid arthritis; Sjögren's syndrome; Type 1 diabetes

\section{Introduction}

In autoimmune polyglandular syndrome (APS) type 3, autoimmune thyroiditis occurs with another organ-specific autoimmune disease, but not with autoimmune adrenalitis [1]. Other systemic autoimmune diseases can include type 1 diabetes mellitus, Sjögren's syndrome, and rheumatoid arthritis. Here, we report a patient with APS type 3 who showed a significant discrepancy between endocrinological

Manuscript accepted for publication July 29, 2011

${ }^{a}$ Department of Internal Medicine, National Center for Global Health and Medicine Kohnodai Hospital, Chiba, Japan

${ }^{\mathrm{b}}$ Clinical Research Center, National Center for Global Health and

Medicine Kohnodai Hospital, Chiba, Japan

${ }^{\mathrm{c} C o r r e s p o n d i n g ~ a u t h o r: ~ H i d e k a t s u ~ Y a n a i, ~ D e p a r t m e n t ~ o f ~ I n t e r n a l ~}$ Medicine, Clinical Research Center, National Center for Global Health and Medicine Kohnodai Hospital, 1-7-1 Kohnodai, Ichikawa, Chiba

272-8516, Japan. Email: dyanai@hospk.ncgm.go.jp

doi: $10.4021 / \mathrm{jmc} 262 \mathrm{w}$ and clinical phenotype and immunological phenotype.

\section{Case Report}

A 53-year-old woman was diagnosed as having autoimmune thyroiditis due to the swelling of thyroid and the positivity for anti-thyroid peroxidase antibody (anti-TPO ab) and antithyroglobulin antibody (anti-TG ab) 25 years ago, however, she did not require thyroxin substitution because her thyroid function test was normal. She was also diagnosed as having Sjögren's syndrome due to dryness of eye and mouth and the positivity for anti-SSA/Ro antibody (anti-SSA/Ro ab) and anti-SSB/La antibody (anti-SSB/La ab) 4 years ago, and she has been treated by cevimeline hydrochloride hydrate. She was referred to our hospital due to hyperglycemia. Her body weight was $62.9 \mathrm{~kg}$ and height $157.4 \mathrm{~cm}$ (BMI $25.4 \mathrm{~kg} /$ $\left.\mathrm{m}^{2}\right)$. Plasma glucose $(335 \mathrm{mg} / \mathrm{dL})$ and HbA1c $(8.8 \%)$ levels were significantly elevated. Although titers of anti-TPO $\mathrm{ab}$ and anti-TG ab were significantly high, serum levels of free triiodothyronine, free thyroxine and thyroid stimulating hormone were within normal range. The titers of both antiglutamic acid decarboxylase antibody (anti-GAD ab) and anti-insulinoma-associated protein-2 antibody (anti-IA-2 ab) were remarkably high, however, the urinary C-peptide level was within normal range (Table 1), suggesting the preserved beta-cell function. Her blood glucose levels were $101-167$ $\mathrm{mg} / \mathrm{dL}$ by using 2 units of insulin glulisine before each meal. Her adrenal function was normal. Although she has been diagnosed as having APS type 3, she was euthyroid and insulin-independent. Further, titer of rheumatoid factor (RF) was remarkably high, however, she did not show arthritis and her serum anti-cyclic citrullinated peptide antibody (anti-CCP ab) and C-reactive protein (CRP) were negative (Table 1).

\section{Discussion}

APS is made up of a group of autoimmune disorders of the endocrine glands [2]. APS shows failure of the glands to produce their hormones. APS type 3 , in contrast to APS type 
Table 1. Clinical, Immunological, and Endocrinological Characteristics of Our Patient with Autoimmune Polyglandular Syndrome Type 3

\begin{tabular}{|c|c|c|c|c|}
\hline Autoantibodies & Titer & Suspected diseases & Endocrinological findings & Symptoms and other data \\
\hline anti-TPO ab & $\begin{array}{l}>600 \mathrm{IU} / \mathrm{ml} \\
(\mathrm{NR}<16)\end{array}$ & Hashimoto's disease & $\begin{array}{l}\text { TSH } 3.62 \mu \mathrm{IU} / \mathrm{mL} \\
(\mathrm{NR}, 0.54-4.26) \\
\text { free } \mathrm{T} 33.51 \mathrm{pg} / \mathrm{mL}\end{array}$ & asymptomatic \\
\hline anti-TG ab & $\begin{array}{l}298 \mathrm{IU} / \mathrm{mL} \\
(\mathrm{NR}<28)\end{array}$ & & $\begin{array}{l}(\mathrm{NR}, 2.39-4.06) \\
\text { free T4 } 1.11 \mathrm{ng} / \mathrm{dL} \\
(\mathrm{NR}, 0.71-1.52)\end{array}$ & \\
\hline anti-GAD ab & $\begin{array}{l}10000 \mathrm{U} / \mathrm{Ml} \\
(\mathrm{NR}<1.5)\end{array}$ & type 1 diabetes & $\begin{array}{l}\text { urinary C-peptide } 70 \mu \mathrm{g} / \text { day } \\
(\mathrm{NR}, 29.2 \text { - 167) }\end{array}$ & $\begin{array}{l}\text { BS } 335 \mathrm{mg} / \mathrm{dL} \\
\mathrm{HbA} 1 \mathrm{c} 8.8 \%\end{array}$ \\
\hline anti-IA-2 ab & $\begin{array}{l}11.0 \mathrm{U} / \mathrm{mL} \\
(\mathrm{NR}<0.4)\end{array}$ & & & \\
\hline anti-SSA/Ro ab & $\begin{array}{l}>500 \mathrm{U} / \mathrm{mL} \\
(\mathrm{NR}<10)\end{array}$ & Sjögren's syndrome & & dry eye and dry mouth \\
\hline anti-SSB/La ab & $\begin{array}{l}139 \mathrm{U} / \mathrm{Ml} \\
(\mathrm{NR}<10)\end{array}$ & & & \\
\hline $\mathrm{RF}$ & $\begin{array}{l}320 \mathrm{IU} / \mathrm{mL} \\
(\mathrm{NR}<20)\end{array}$ & rheumatoid arthritis & & $\begin{array}{l}\text { asymptomatic } \\
\text { anti-CCP ab (-) } \\
\text { CRP (-) }\end{array}$ \\
\hline
\end{tabular}

ab: antibody; BS: blood sugar; CCP: cyclic citrullinated peptide; CRP: C-reactive protein; GAD: glutamic acid decarboxylase; IA-2: insulinoma-associated protein-2; NR: normal range; RF: rheumatoid arthritis; T3: triiodothyronine; T4: thyroxine; TG: thyroglobulin; TPO: thyroid peroxidase; TSH: thyroid stimulating hormone.

Table 2. Major Component Diseases and Minor Autoimmune Diseases in Each Type of Autoimmune Polyglandular Syndrome [1]

\begin{tabular}{|c|c|c|c|c|}
\hline & Type 1 & Type 2 & Type 3 & Type 4 \\
\hline \multirow[t]{4}{*}{$\begin{array}{l}\text { major component } \\
\text { diseases }\end{array}$} & Addison disease & Addison disease & $\begin{array}{l}\text { thyroid autoimmune } \\
\text { diseases }\end{array}$ & Addison disease \\
\hline & hypoparathyroidism & $\begin{array}{l}\text { thyroid autoimmune } \\
\text { diseases }\end{array}$ & 3A-type 1 diabetes & \\
\hline & candidiasis & type 1 diabetes & 3B-pernicious anemia & \\
\hline & & & $\begin{array}{l}\text { 3C-vitiligo, alopecia, } \\
\text { other organ-specific } \\
\text { autoimmune diseases }\end{array}$ & \\
\hline \multirow{8}{*}{$\begin{array}{l}\text { minor autoimmune } \\
\text { diseases }\end{array}$} & type 1 diabetes & myasthenia gravis & Celiac disease & gonadal failure \\
\hline & gonadal failure & gonadal failure & malabsorption & malabsorption \\
\hline & pernicious anemia & vitiligo & gonadal failure & hypophysitis \\
\hline & malabsorption & alopecia & sarcoidosis & $\begin{array}{l}\text { autoimmune } \\
\text { hepatitis }\end{array}$ \\
\hline & vitiligo & pernicious anemia & Sjögren's syndrome & pernicious anemia \\
\hline & alopecia & & rheumatoid arthritis & myasthenia gravis \\
\hline & & & myasthenia gravis & vitiligo \\
\hline & & & & alopecia \\
\hline
\end{tabular}


1 and 2 [3], does not involve the adrenal dysfunction (Table 2) [1]. In APS type 3, autoimmune thyroiditis occurs with another organ-specific autoimmune disease (Table 2) [1]. APS type 3 can be further classified into the 3 subgroups. Autoimmune thyroiditis with type 1 diabetes mellitus which developed in my patient is categorized as APS type 3A. APS type 3 is associated with organ-specific autoimmune diseases such as Celiac disease and myasthenia gravis, and systemic autoimmune diseases such as sarcoidosis, Sjögren's syndrome and rheumatoid arthritis [1].

Most patients with APS type 3 show abnormal thyroid function and decreased insulin secretion [4-6]. We experience a very rare APS type 3 middle-aged patient who showed normal thyroid and preserved beta-cell function in spite of the existence of remarkably high titer of autoantibodies (anti-TPO ab, anti-TG ab, anti-GAD ab and anti-IA-2 ab). Further, she presented with symptoms of Sjögren's syndrome (dryness of eye and mouth) and has been treated, showing the positivity for anti-SSA/Ro antibody and anti-SSB/La antibody. However, in spite of remarkably high titer of RF, she did not show arthritis and her serum anti-CCP ab and CRP were negative (Table 1).

APS is diverse, and its diversity is a characteristic that is both clinically important and very informative to understand its underlying basic immunological mechanisms and features [7-9]. An accumulation of similar cases to our patient may prompt us to understand the pathogenesis for APS.

\section{References}

1. Betterle C, Dal Pra C, Mantero F, Zanchetta R. Autoim- mune adrenal insufficiency and autoimmune polyendocrine syndromes: autoantibodies, autoantigens, and their applicability in diagnosis and disease prediction. Endocr Rev. 2002;23(3):327-364.

2. Kahaly GJ. Polyglandular autoimmune syndromes. Eur J Endocrinol. 2009;161(1):11-20.

3. Neufeld M, Blizzard RM. Polyglandular autoimmune disease. In: Pinchera A, Doniach D, Fenzi DF, Baschieri L, eds. Autoimmune aspects of endocrine disorders. London, UK: Academic Press; 1980:357-365.

4. Hummel M, Banholzer P, Rabl W, Ziegler AG. Organospecific lymph node enlargement in autoimmune polyglandular syndrome. Diabetes Care. 1998;21(9):15731574.

5. Shimomura H, Nakase $Y$, Furuta $H$, Nishi M, Nakao T, Hanabusa T, Sasaki H, et al. A rare case of autoimmune polyglandular syndrome type 3. Diabetes Res Clin Pract. 2003;61(2):103-108.

6. Oki K, Yamane K, Koide J, Mandai K, Nakanishi S, Fujikawa R, Kohno N. A case of polyglandular autoimmune syndrome type III complicated with autoimmune hepatitis. Endocr J. 2006;53(5):705-709.

7. Eisenbarth GS, Gottlieb PA. Autoimmune polyendocrine syndromes. N Engl J Med. 2004;350(20):2068-2079.

8. Neufeld M, Maclaren NK, Blizzard RM. Two types of autoimmune Addison's disease associated with different polyglandular autoimmune (PGA) syndromes. Medicine (Baltimore). 1981;60(5):355-362.

9. Ahonen P, Myllarniemi S, Sipila I, Perheentupa J. Clinical variation of autoimmune polyendocrinopathy-candidiasis-ectodermal dystrophy (APECED) in a series of 68 patients. N Engl J Med. 1990;322(26):1829-1836. 